\title{
Universal scaling for the quantum Ising chain with a classical impurity
}

\author{
Tony J. G. Apollaro, ${ }^{1,2, *}$ Gianluca Francica, ${ }^{3,4}$ Domenico Giuliano, ${ }^{3,4}$ Giovanni Falcone, ${ }^{3,4}$ \\ G. Massimo Palma, ${ }^{5,6}$ and Francesco Plastina ${ }^{3,4}$ \\ ${ }^{1}$ Quantum Technology Lab, Dipartimento di Fisica, Università degli Studi di Milano, 20133 Milano, Italy \\ ${ }^{2}$ Centre for Theoretical Atomic, Molecular and Optical Physics, School of Mathematics and Physics, Queen's University Belfast, \\ Belfast BT7 1NN, United Kingdom \\ ${ }^{3}$ Dipartimento di Fisica, Università della Calabria Arcavacata di Rende I-87036, Cosenza, Italy \\ ${ }^{4}$ I.N.F.N., Gruppo collegato di Cosenza, Arcavacata di Rende I-87036, Cosenza, Italy \\ ${ }^{5}$ Dipartimento di Fisica e Chimica, Università degli Studi di Palermo, via Archirafi 36, I-90123 Palermo, Italy \\ ${ }^{6}$ NEST, Istituto Nanoscienze-CNR, 56127 Pisa, Italy \\ (Received 4 July 2017; published 30 October 2017)
}

\begin{abstract}
We study finite-size scaling for the magnetic observables of an impurity residing at the end point of an open quantum Ising chain with transverse magnetic field, realized by locally rescaling the field by a factor $\mu \neq 1$. In the homogeneous chain limit at $\mu=1$, we find the expected finite-size scaling for the longitudinal impurity magnetization, with no specific scaling for the transverse magnetization. At variance, in the classical impurity limit $\mu=0$, we recover finite scaling for the longitudinal magnetization, while the transverse one basically does not scale. We provide both analytic approximate expressions for the magnetization and the susceptibility as well as numerical evidences for the scaling behavior. At intermediate values of $\mu$, finite-size scaling is violated, and we provide a possible explanation of this result in terms of the appearance of a second, impurity-related length scale. Finally, by going along the standard quantum-to-classical mapping between statistical models, we derive the classical counterpart of the quantum Ising chain with an end-point impurity as a classical Ising model on a square lattice wrapped on a half-infinite cylinder, with the links along the first circle modified as a function of $\mu$.
\end{abstract}

DOI: 10.1103/PhysRevB.96.155145

\section{INTRODUCTION}

Quantum phase transitions embody one of the most striking collective behaviors of many-body systems [1]. At variance with thermal fluctuation-induced phase transitions, a quantum phase transition in a many-body system is typically triggered by quantum fluctuations and, therefore, it can take place even at zero temperature, once a system parameter (say $h$ ) is tuned across its critical value $\left(h_{c}\right)$. In analogy with thermal ones, the classification of quantum phase transitions relies upon the Eherenfest-Landau scheme, by which one defines the order of a transition as that of the lowest derivative of the pertinent free-energy functional, showing a discontinuity at the critical point (see Ref. [2] for a review on the subject). A milestone in the construction of a systematic theory of the phase transitions is the concept of universality, stating that all the physical systems sharing the same dimensionality, symmetry of the order parameter, and range of the interaction are expected to behave alike, close to a phase transition and are said to belong to the same universality class $[3,4]$. Universality is a consequence of the divergence of the correlation length $\xi$ at the critical point [4]. Indeed, near a second-order quantum phase transition, the growth of $\xi$ makes it the only relevant length scale of the system, and makes the microscopic details of the system irrelevant. Furthermore, it implies the scaling of physical quantities as power laws of $\left(h_{c}-h\right)$, with critical exponents that take the same values throughout the whole universality class [5]. In addition, close to a critical point, the algebraic divergence of $\xi$ implies scale invariance of the system; that is, general physical quantities behave as powers of control parameters times some scaling functions of

*Corresponding author: tony.apollaro@gmail.com dimensionless ratios such as, for instance, energy/(Boltzmann constant times) temperature, etc. [4,6]. An astonishing consequence of such a prediction is that observables such as magnetization, susceptibility, correlation length and time, specific heat, as well as quantities which are not observables in the quantum-mechanical sense, such as entanglement [7-9], Schmidt gap [10], irreversible work [11], all exhibit a scaling behavior according to a set of critical indexes which define the universality class the model belongs to.

Due to the recent progress in designing and fabricating quantum devices with engineered properties, a remarkable interest has been triggered in the physics of impurities in critical or quasicritical systems [12]. As an example, local impurities have been proposed to improve the efficiency in quantum state transfer protocols $[13,14]$.

When impurities are realized in a critical system (the "bulk"), the lack of reference (energy or length) scales in the bulk allows for the emergence of dynamically generated impurity-related scales, such as the Kondo temperature, or the Kondo length, in the case of magnetic impurities antiferromagnetically coupled to a bulk of itinerant electrons $[15,16]$, or of lattice quantum spin systems [17,18], or the healing length, in the case of tunneling between interacting electronic systems in one spatial dimension [19]. Typically, the impurity dynamics affects bulk quantities (such as the conductance, or the spin susceptibility), and, in turn, it can be probed by looking at the bulk response through suitably designed devices. Recently, impurity-induced dynamics has been investigated, e.g., in Josephson junction networks [20-23], quantum spin chains [24-29], and cold fermion gases [30-33].

While there are a remarkably large number of possible bulk effects induced by the impurity dynamics, in this paper we take a complementary point of view; namely, we rather 
look at the effects that a critical many-body bulk system has on the impurity. In fact, several proposals have recently put forward to engineer fully controllable quantum objects as "quantum probes" of many-body condensed matter systems (see, e.g., Refs. [34-38]). In the specific context of a quantum impurity embedded within a bulk system close to a quantum phase transition, either the impurity generates a dynamic scale that rules the scaling of its observables or, if this does not happen, the impurity-related observables scale with exponents that are directly linked to the system ones. In the context of thermal phase transitions, this effect has been demonstrated, e.g., for the surface magnetization in inhomogeneous two-dimensional Ising lattices [39]. Due to the remarkable correspondence between $(d+1)$-dimensional classical systems and quantum $d$-dimensional ones [40,41], one expects a similar behavior to emerge for a quantum impurity embedded within one-dimensional critical systems, namely, that the scaling in the bulk implies some sort of scaling in the impurity observables, as well $[42,43]$.

To spell out the consequences of the bulk scaling on observable quantities of a boundary impurity, here we study the open, one-dimensional, quantum Ising model in a transverse field $h$ containing a side impurity. Since the seminal works dating back to the 1960s [44,45], the transverse field Ising model has received a great attention in the literature; its critical indexes are since long well known [46,47], as well as experimentally verified (see Ref. [48] and references therein). The effects of disorder and/or impurities have been considered as well, unveiling quite a rich phenomenology that ranges from the so-called Griffiths-McCoy singularities [49] to the rounding of a quantum phase transition [50] (for an up-to-date review of theoretical and experimental aspects, see Ref. [51]).

In this paper, we consider a side impurity at the first site of the Ising chain, realized with a local transverse magnetic field equal to a fraction of the bulk one, $\mu h$ with $0 \leqslant \mu \leqslant 1$. At $\mu=1$, our model reduces back to the homogeneous one with open boundary conditions. In contrast, for $\mu=0$, we can explore the physics of a classical impurity (see Ref. [52]). As outlined there, a classical impurity gives rise to a twofold degeneracy for the spectrum of the whole system Hamiltonian. We show here that such an emerging degeneracy results in the scaling behavior of the impurity observables close to the bulk quantum phase transition. Specifically, working at zero temperature, we will perform a finite-size scaling analysis of the physical properties of the impurity and derive the corresponding scaling exponents. In particular, we will investigate the finite-size scaling behavior of the impurity magnetization by looking at both its longitudinal and transverse components (directed along the coupling axis and along the applied magnetic field, respectively).

Close to the homogeneous chain limit $(\mu \sim 1)$, we recover the finite-size scaling of the longitudinal magnetization, which is consistent with the behavior of the edge magnetization in the two-dimensional classical model [39]. At the same time, the transverse magnetization shows no particular scaling properties. On the other hand, when moving towards the classical impurity limit $(\mu \rightarrow 0)$, we find the emergence of finite-size scaling in the transverse magnetization, as well. While this appears to be an already remarkable finding per se, it becomes particularly relevant when interpreted along the results obtained in Ref. [53] where the energy spectrum is obtained for the Hamiltonian of the inhomogeneous transverse field Ising model.

The transverse field Ising model is known to exhibit a quantum phase transition between an ordered phase and a paramagnetic one [1]. Both phases are characterized by an excitation spectrum with a finite-energy gap, with spectra that appear quite similar to each other, except for the appearance of a subgap mode in the ordered phase, which eventually evolves towards an actual zero-energy excitation as the size of the system increases. It is exactly the appearance of the subgap mode that determines the scaling behavior of the longitudinal impurity magnetization in the ordered phase [39] and, by converse, its absence which determines the scaling to zero of the longitudinal magnetization in the thermodynamic limit. The analogous contribution to the transverse magnetization is, in general, overwhelmed by the contributions from the modes with energy above the gap, which yields no particular scaling behavior. In our inhomogeneous case, when $\mu \rightarrow 0$, two important things happen: first, an additional subgap mode emerges in the paramagnetic region and, second, the contribution to the transverse magnetization from above-thegap modes shrinks to zero, thus providing the transverse magnetization itself with an order-parameter-like behavior analogous to that of the longitudinal magnetization, but now in the paramagnetic phase, rather than in the ordered one. As a result, the behavior of the transverse magnetization close to the quantum phase transition can be directly linked to the emergence of such a subgap mode in the paramagnetic region, which is the second, remarkable conclusion of our work.

The paper is organized as follows: In Sec. II, for the sake of self-completeness, we briefly recap the main results for the Ising model with open boundary conditions and a single edge impurity reported in Ref. [53]; in Sec. III, we report the critical exponents of the impurity magnetic observables, which are then used in Sec. IV to obtain the predicted data collapse. Finally in Sec. V, the universality hypothesis is checked by verifying that the scaling exponents remain the same also for the $X Y$ model. Going along the correspondence between $d$-dimensional quantum models and $(d+1)$-dimensional classical statistical systems, in Sec. VI we derive the classical, two-dimensional analog of the transverse field Ising model with an end-point impurity. Finally, in Sec. VII we provide our main conclusions.

\section{IMPURITY MODEL HAMILTONIAN}

We model the quantum impurity by rescaling the transverse magnetic field $h$ at one end point of an $N$-site quantum Ising chain to $\mu h$, with $\mu$ being a dimensionless parameter. Accordingly, our model Hamiltonian $H_{\mu}$ can be regarded as a special case of the transverse field Ising model Hamiltonian in a nonuniform transverse magnetic field $h_{n}$, that is,

$$
\hat{H}_{\mu}=-J \sum_{n=1}^{N-1} \hat{\sigma}_{n}^{x} \hat{\sigma}_{n+1}^{x}-J \sum_{n=1}^{N} h_{n} \hat{\sigma}_{n}^{z},
$$

with $\hat{\sigma}_{n}^{\alpha}(\alpha=x, y, z)$ being the Pauli matrices corresponding to (two times) the components of a spin- $\frac{1}{2}$ quantum operator at site $n$ of the chain, $J$ being an overall energy scale (used as 


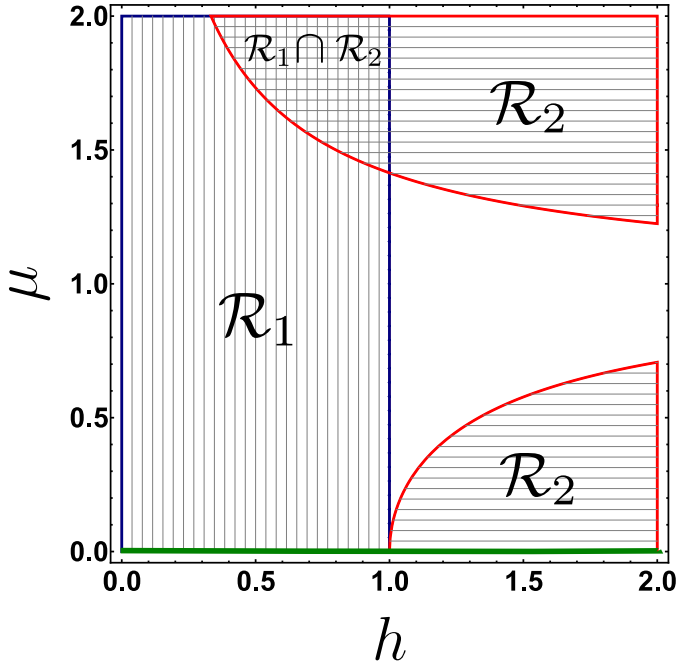

FIG. 1. Distribution of the discrete modes $\Lambda_{n}(n=1,2)$ in the $h, \mu$ plane. In the region $\mathcal{R}_{1}$ (vertical lines), only the mode $\Lambda_{1}$ is present, while in the region $\mathcal{R}_{2}$ (horizontal lines), there is only the mode $\Lambda_{2}$, either below or above the band in the two subregions with $\mu<1$ or $\mu>1$, respectively. Both discrete modes are present in the intersection of $\mathcal{R}_{1}$ and $\mathcal{R}_{2}$. On the solid green line $(\mu=0$ with $h \geqslant 1)$, mode 2 becomes an actual zero mode at any finite $N\left(\Lambda_{2}=0\right)$ and the impurity becomes classical [52]. Finally, only quasicontinuous modes are present in the white region.

a reference scale henceforth), and $h_{n}=h\left(1-\delta_{n, 1}\right)+\mu h \delta_{n, 1}$. The model in Eq. (1) undergoes a quantum phase transition if the magnetic field is set at the critical value $h_{c}=1$. In the following, we will discuss the impurity physics for $h \sim h_{c}$.

By the standard Jordan-Wigner representation of the spin- $\frac{1}{2}$ operators in terms of lattice spinless fermion operators $\left\{c_{n}, c_{n}^{\dagger}\right\}$, giving [54] $\hat{\sigma}_{n}^{x}=\left[c_{n}+c_{n}^{\dagger}\right] e^{i \pi \sum_{r=1}^{n-1} c_{r}^{\dagger} c_{r}}$ and $\hat{\sigma}_{n}^{z}=1-2 c_{n}^{\dagger} c_{n}$, $\hat{H}_{\mu}$ is traded for an exactly solvable quadratic fermion Hamiltonian. The derivation of the eigenvalues of $\hat{H}_{\mu}$ and of the corresponding eigenmodes is discussed in detail in Ref. [53]. Here, for the sake of the presentation, we just review the main results. By introducing the Bogoliubov-de Gennes quasiparticle operators $\left\{\eta_{q}, \eta_{q}^{\dagger}\right\}$, related to the $\left\{c_{n}, c_{n}^{\dagger}\right\}$ by the Bogoliubov-Valatin transformations

$$
\begin{aligned}
& \hat{\eta}_{q}=\sum_{n}\left\{\frac{\psi_{q n}+\phi_{q n}}{2} \hat{c}_{n}+\frac{\psi_{q n}-\phi_{q n}}{2} \hat{c}_{n}^{\dagger}\right\}, \\
& \hat{\eta}_{q}^{\dagger}=\sum_{n}\left\{\frac{\psi_{q n}-\phi_{q n}}{2} \hat{c}_{n}+\frac{\psi_{q n}+\phi_{q n}}{2} \hat{c}_{n}^{\dagger}\right\},
\end{aligned}
$$

we can recast $\hat{H}_{\mu}$ in the form

$$
\hat{H}_{\mu}=\sum_{\kappa} \Lambda_{\kappa} \hat{\eta}_{\kappa}^{\dagger} \hat{\eta}_{\kappa}+\chi_{1} \Lambda_{1} \hat{\eta}_{1}^{\dagger} \hat{\eta}_{1}+\chi_{2} \Lambda_{2} \hat{\eta}_{2}^{\dagger} \hat{\eta}_{2} .
$$

In Eq. (3), $\kappa$ labels the quasicontinuous modes, with energy larger than the single-quasiparticle bulk gap $\Delta_{m}=|1-h|$ : these are typically parametrized in terms of the "angles" $\theta_{\kappa}$ which solve the secular equations (A12) of Ref. [53]. In addition, $\hat{H}_{\mu}$ includes contributions from the two discrete modes $\eta_{1}, \eta_{2}$, which, depending on the values of $h$ and $\mu$, may appear at energies lying within $\Delta_{m}$. On labeling with $\mathcal{R}_{n}$ the region in parameter space where mode $n=1,2$ exists, we see that $\mathcal{R}_{1}$ is the ferromagnetic region $h \leqslant 1$, while $\mathcal{R}_{2}=\{(h, \mu)$ : $(\forall h \wedge|\mu|>\sqrt{1+1 / h}) \vee(h>1 \wedge|\mu|<\sqrt{1-1 / h})\}\left(\mathcal{R}_{1,2}\right.$ are depicted in Fig. 1; the details about their construction are provided in Ref. [53]). Furthermore, in Eq. (3), we used $\chi_{1}=\Theta(1-h)$ and $\chi_{2}$ to denote the characteristic functions of $\mathcal{R}_{1}$ and $\mathcal{R}_{2}$, respectively, so that the corresponding fermion mode $n=1(n=2)$ is absent if $h, \mu$ are taken outside $\mathcal{R}_{1}$ $\left(\mathcal{R}_{2}\right)$. The wave functions $\psi_{q n}, \phi_{q n}$ entering Eq. (2), derived in Ref. [53], are reported here in Table I, where we list the energy eigenvalues of $\hat{H}_{\mu}$ and the corresponding eigenstates (in the $N \rightarrow \infty$ limit).

\section{SCALING ANALYSIS OF THE TRANSVERSE AND LONGITUDINAL MAGNETIZATION IN THE THERMODYNAMIC LIMIT}

In this section, we investigate the scaling of the transverse and longitudinal impurity magnetization in the vicinity of the bulk quantum phase transition $\left(h \sim h_{c}=1\right)$ in the thermodynamic limit $N \rightarrow \infty$. In addition, we also discuss the scaling of the impurity transverse susceptibility close to the critical point.

\section{A. Local transverse magnetization}

The transverse magnetization at site $n$ of the transverse field Ising model is defined as the average value of the $z$ component of the spin operator at that site $\left\langle\hat{\sigma}_{n}^{z}\right\rangle$. Using the Jordan-Wigner transformations and inverting the BogoliubovValatin transformations in Eqs. (2), setting $v_{q n}=\frac{\psi_{q n}-\phi_{q n}}{2}$, one obtains

$$
\left\langle\hat{\sigma}_{n}^{z}\right\rangle=1-2\left\langle c_{n}^{\dagger} c_{n}\right\rangle=1-2 \sum_{q} v_{q n}^{2} .
$$

When computing Eq. (4) for $n=1$ (transverse impurity magnetization), we see that $\left\langle\hat{\sigma}_{1}^{z}\right\rangle$ consists of various contributions, due to the quasicontinuous modes $\lambda_{\kappa}$ and to the discrete modes

TABLE I. Expressions for the energy eigenvalues and the $\{\psi, \phi\}$ matrix elements in the $(h, \mu)$ plane [see Eq. (A12) of Ref. [53] for the definition of the parameter $\left.\theta_{\kappa}\right]$.

\begin{tabular}{lccc}
\hline \hline$(h, \mu)$ & $\Lambda$ & $\psi$ & $\phi$ \\
\hline$\forall(h, \mu)$ & $\Lambda_{\kappa}=2 \sqrt{1+h^{2}-2 h \cos \theta_{\kappa}}$ & $\psi_{n}\left(\theta_{\kappa}\right)=\sqrt{\frac{2}{N}} \frac{\sin \left(n \theta_{\kappa}\right)+\left(\mu^{2}-1\right) h \sin \left[(n-1) \theta_{\kappa}\right]}{\sqrt{1+\left(\mu^{2}-1\right)^{2} h^{2}+2 h\left(\mu^{2}-1\right) \cos \theta_{\kappa}}}$ & $\phi_{n}\left(\theta_{\kappa}\right)=\frac{2 h}{\Lambda_{\kappa}} \psi_{n}\left(\theta_{\kappa}\right)-\frac{2(\mu+1) h \delta_{n 1}}{\Lambda_{\kappa}} \psi_{1}\left(\theta_{\kappa}\right)-\frac{2\left(1-\delta_{n 1}\right)}{\Lambda_{\kappa}} \psi_{n-1}\left(\theta_{\kappa}\right)$ \\
$\mathcal{R}_{1}$ & $\Lambda_{1}=\frac{2|\mu|\left(1-h^{2}\right) h^{N}}{\sqrt{\left|1+\left(\mu^{2}-1\right) h^{2}\right|}}$ & $\psi_{n}^{(1)}=\sqrt{1-h^{2}}\left(h^{N-n}-\frac{\mu^{2} h^{N+n}}{1+\left(\mu^{2}-1\right) h^{2}}\right)$ & $\phi_{n}^{(1)}=\frac{\sqrt{1-h^{2}} \sqrt{\left|1+\left(\mu^{2}-1\right) h^{2}\right|}}{|\mu| h\left(1+\left(\mu^{2}-1\right) h^{2}\right]}\left\{1-(\mu+1)\left[\delta_{n 1}+\left(1-\delta_{n 1}\right)(1-\mu)\right]\right\} h^{n}$ \\
$\mathcal{R}_{2}$ & $\Lambda_{2}=2|\mu| \sqrt{\frac{1+\left(\mu^{2}-1\right) h^{2}}{\left(\mu^{2}-1\right)}}$ & $\psi_{n}^{(2)}=\frac{(-1)^{n} h^{-n} \sqrt{\left(\mu^{2}-1\right)^{2} h^{2}-1}}{\left(\mu^{2}-1\right)^{n}}$ & $\phi_{n}^{(2)}=\frac{2 h}{\Lambda^{(2)}} \psi_{n}^{(2)}-\frac{2(\mu+1) h \delta_{n 1}}{\Lambda^{(2)}} \psi_{1}^{(2)}-2 \frac{1-\delta_{n 1}}{\Lambda^{(2)}} \psi_{n-1}^{(2)}$ \\
\hline \hline
\end{tabular}



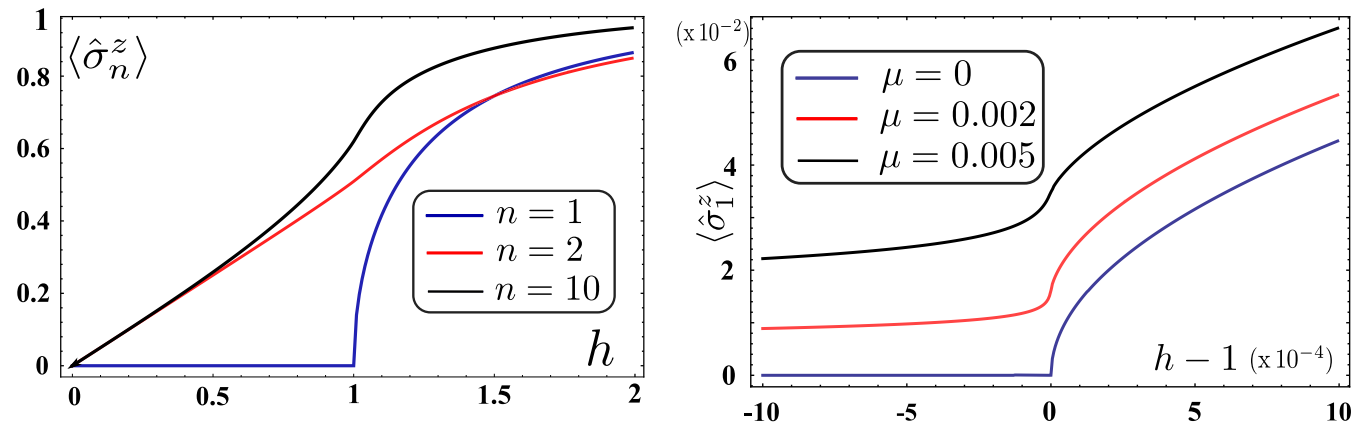

FIG. 2. Left panel: transverse magnetization $\left\langle\hat{\sigma}_{n}^{z}\right\rangle$ in the thermodynamic limit $(N \rightarrow \infty)$ as a function of the magnetic field $h$, as $\mu \rightarrow 0$, for the impurity spin $n=1$ and, for comparison, for spins $n=2$ and 10 as well. Apparently, only $\left\langle\hat{\sigma}_{n}^{z}\right\rangle$ behaves as a continuous (dis)order parameter as $h \rightarrow h_{c}$. Right panel: transverse impurity magnetization $\left\langle\hat{\sigma}_{n}^{z}\right\rangle$ (in the limit $N \rightarrow \infty$ ), near $h_{c}$ and for $\mu=0,0.002,0.005$. The steplike behavior holds only for $\mu=0$.

$\Lambda_{1,2}$. On singling out these three terms, one may write

$$
\left\langle\hat{\sigma}_{1}^{z}\right\rangle=\varphi_{\mathrm{cm}}+\varphi_{1}+\varphi_{2},
$$

with

$$
\begin{aligned}
\varphi_{\mathrm{cm}} & =-\frac{4 \mu}{N} \sum_{\kappa}\left\{\frac{\sin ^{2} \theta_{\kappa}}{\Lambda_{\kappa}\left[1+\left(\mu^{2}-1\right)^{2} h^{2}+2 h\left(\mu^{2}-1\right) \cos \theta_{\kappa}\right]}\right\} \\
\varphi_{1} & =-2\left[\frac{h^{N} \operatorname{sgn}(\mu)}{2 h}\right]\left[\frac{\left(1-h^{2}\right)^{2}}{\sqrt{1+\left(\mu^{2}-1\right) h^{2}}}\right] \operatorname{sgn}\left[1+\left(\mu^{2}-1\right) h^{2}\right] \\
\varphi_{2} & =-2\left[\frac{\operatorname{sgn}(\mu)}{h\left(\mu^{2}-1\right)^{\frac{3}{2}}}\right]\left[\frac{\left(\mu^{2}-1\right)^{2} h^{2}-1}{\sqrt{\left(\mu^{2}-1\right) h^{2}+1}}\right] .
\end{aligned}
$$

Equations (5) and (6) allow us to separately discuss the large- $N$ limit of the various contributions to $\left\langle\hat{\sigma}_{1}^{z}\right\rangle$ close to the quantum phase transition. First of all, we note that, in the homogeneous chain limit $(\mu \rightarrow 1), \varphi_{2}$ does not contribute as the mode $\Lambda_{2}$ is not present in the spectrum on the horizontal line $\mu=1$ in the parameter space (see Fig. 1). In this case, one simply obtains

$$
\left\langle\hat{\sigma}_{1}^{z}\right\rangle=-h^{N-1}\left(1-h^{2}\right)^{2}-\frac{4}{N} \sum_{\kappa}\left\{\frac{\sin ^{2} \theta_{\kappa}}{\Lambda_{\kappa}}\right\} .
$$

As $N \rightarrow \infty$, the contribution from the discrete mode $\Lambda_{1}$ is exponentially suppressed, and one obtains

$$
\left\langle\hat{\sigma}_{1}^{z}\right\rangle \rightarrow-\frac{2}{\pi} \int_{0}^{\pi} d \theta\left\{\frac{\sin ^{2} \theta}{\sqrt{1+h^{2}-2 h \cos \theta}}\right\},
$$

which is the standard result for the homogeneous transverse field Ising model.

At variance, when $\mu \rightarrow 0$, the discrete mode $\Lambda_{2}$ emerges with vanishing energy at any finite $N$. This corresponds to the impurity at $n=1$ becoming classical [52]. Accordingly, the Hamiltonian spectrum becomes twofold degenerate at any finite $N$ and one may always construct a conserved operator commuting with $\hat{H}_{\mu=0}$, but anticommuting with the Jordan-Wigner total fermion parity operator (see Ref. [52] for a detailed discussion of this point). Taking the $N \rightarrow \infty$ limit of Eq. (6), one eventually obtains

$$
\begin{aligned}
\left\langle\hat{\sigma}_{1}^{z}\right\rangle= & 1-2\left[\int_{0}^{\pi} d \theta v_{1}^{2}(\theta)+\Theta(h-1) \Theta\left(x^{+}\right) \Theta\left(y^{+}\right)\left(v_{1}^{(2)}\right)^{2}\right. \\
& \left.+\Theta(1-h)\left(\left[\Theta\left(y^{-}\right)+\Theta\left(x^{-}\right)\right]\left(v_{1}^{(2)}\right)^{2}+\left(v_{1}^{(1)}\right)^{2}\right)\right],
\end{aligned}
$$

with $x^{ \pm}=\mu h+\sqrt{h(h \mp 1)}, y^{ \pm}=-\mu h+\sqrt{h(h \mp 1)}$, and $\Theta(x)$ being the Heaviside step function. As $\mu \rightarrow 0$, Eq.(9) yields

$$
\lim _{\mu \rightarrow 0^{ \pm}}\left\langle\hat{\sigma}_{1}^{z}\right\rangle=\mp \frac{(h+1)^{\frac{1}{2}}(h-1)^{\frac{1}{2}}}{h} \Theta(h-1) .
$$

From Eq. (10), we see that the transverse magnetization has a power-law behavior near $h_{c}$. Indeed, as $h \rightarrow h_{c}$, one has $\left\langle\hat{\sigma}_{1}^{z}\right\rangle \simeq 2(h-1)^{\tilde{\beta}}$, with critical exponent $\tilde{\beta}=\frac{1}{2}$. Moreover, Eq. (10) hints towards a singular behavior of the $\mu \rightarrow$ 0 impurity transverse susceptibility $\chi_{z}^{(1)}=\frac{\partial\left\langle\hat{\sigma}_{1}\right\rangle}{\partial h}$ at $h \sim h_{c}$. Remarkably, such a singular behavior of $\chi_{z}^{(1)}$ only holds in the $\mu \rightarrow 0$ limit (see Fig. 2). Note that the transverse magnetization does not show any singularity in the homogeneous transverse field Ising model $(\mu=1)$, regardless of whether the boundary conditions are open or periodic. Now, from Eq. (10), we see that, instead, as $\mu \rightarrow 0,\left\langle\hat{\sigma}_{1}^{z}\right\rangle$ acquires a behavior similar to what one would expect from an order parameter, except that it takes a nonzero ground-state expectation value in the disordered paramagnetic phase $\left(h>h_{c}=1\right)$ rather than in the ordered ferromagnetic one. This behavior is, in fact, a direct consequence of the emergence of the discrete mode $\Lambda_{2}$ and of the fact that it becomes a zero mode as $\mu \rightarrow 0$. The key point is that, for all $\mu$, one has $\left[\hat{H}_{\mu}, \hat{P}\right]=0$, where $\hat{P}$ is the parity operator defined as $\hat{P} \hat{\sigma}_{n}^{x, y} \hat{P}=-\hat{\sigma}_{n}^{x, y}$ and $\hat{P} \hat{\sigma}_{n}^{z} \hat{P}=\hat{\sigma}_{n}^{z}$. In fermionic coordinates, $\hat{P}$ can be regarded as the operator that changes the total fermion parity of a state,

$$
\hat{P}=\prod_{n=1}^{N} \hat{\sigma}_{n}^{z}=\prod_{n=1}^{N} e^{i \pi c_{n}^{\dagger} c_{n}},
$$

with the middle term of Eq. (11) yielding the realization of $\hat{P}$ in spin coordinates, while the last term that for Jordan-Wigner fermions. By construction, one has $\hat{P} \eta_{q}=-\eta_{q} \hat{P}$, where $\eta_{q}$ 
is any one of the fermion operators in Eq. (2). Therefore, the emergence of a zero-energy $\eta_{q}$ mode is enough to ensure the twofold degeneracy of the spectrum of $\hat{H}_{\mu}$. As it can be readily inferred from Table I, in a homogeneous chain $(\mu=1)$, this only happens for $h<1$ and for $N \rightarrow \infty$, where the onset of the degeneracy implies a twofold-degenerate ground state for the system and, accordingly, the possibility for $\hat{\sigma}_{1}^{x}$ to acquire a nonzero ground-state expectation value, and to act as an order parameter for the ferromagnetic phase.

In few words, in order to recover a nonzero ground-state expectation value for a certain operator and use it as a nonzero order parameter to characterize a given phase, one needs a degenerate ground state to emerge in that phase. This is not the case for the paramagnetic phase of the homogeneous transverse field Ising model $(\mu=1)$ which, indeed, does not show any nonzero order parameter. On the contrary, when $\mu \rightarrow 0$, from Eq. (1) one obtains $\left[\hat{\sigma}_{1}^{x}, \hat{H}_{\mu=0}\right]=0$. Combining this last identity with the observation that $\hat{P} \hat{\sigma}_{x}^{1}=-\hat{\sigma}_{x}^{1} \hat{P}$, we eventually find two operators, both commuting with $\hat{H}_{\mu=0}$, but not commuting with each other. This is enough to ensure the twofold spectral degeneracy of $\hat{H}_{\mu}$, which is ultimately consistent with a nonzero ground-state expectation value $\left\langle\hat{\sigma}_{1}^{z}\right\rangle$ playing the role of a characteristic parameter for such a phase. Remarkably, in this classical impurity limit, the degeneracy emerges at any finite $N$, without the need of the thermodynamic limit [52]. In Jordan-Wigner fermion coordinates, the twofold spectral degeneracy at $\mu=0$ occurs when the discrete mode $\eta_{2}$ becomes gapless: it corresponds to the zero-mode operator in Eq. (14) of Ref. [52], adapted to the case of a classical impurity at the end point of a "semi-infinite" chain. Another important point to stress here is that, while in the ferromagnetic phase one gets $\left\langle\hat{\sigma}_{n}^{x}\right\rangle \neq 0$ for all $n$, both at the boundary and in the bulk of the transverse field Ising model (see Fig. 2), on the contrary, as $\mu \rightarrow 0$, only $\left\langle\hat{\sigma}_{1}^{z}\right\rangle$ behaves like a (dis)order parameter for the paramagnetic phase, as $\left\langle\hat{\sigma}_{n}^{z}\right\rangle$ for $n>1$ does not become zero when entering the ferromagnetic phase $h<h_{c}$.

As for the impurity transverse susceptibility, for $N \rightarrow \infty$ and for $\mu \rightarrow 0$ one obtains $\chi_{z}^{(1)}=\frac{\Theta(h-1)}{2 h^{2} \sqrt{h^{2}-1}}$. Thus, at the quantum phase transition, $\chi_{z}^{(1)}$ exhibits an algebraic divergence ruled by the critical exponent $\tilde{\alpha}=\frac{1}{2}$, at variance with what happens to the transverse susceptibility in the bulk, which, at the quantum phase transition, diverges only logarithmically, hence with critical exponent $\alpha=0$.

\section{B. Local longitudinal magnetization}

To discuss the behavior of the longitudinal magnetization of the impurity $\left\langle\hat{\sigma}_{1}^{x}\right\rangle$ in the thermodynamic limit, one has to consider that, as discussed before, while $\hat{P}$ is always a symmetry of $\hat{H}_{\mu}$, the twofold ground-state degeneracy is only recovered as $N \rightarrow \infty$, if $\mu \neq 0$. Therefore, in performing the calculation for finite- $N$ systems first, and then letting $N \rightarrow \infty$ (as we do below, when discussing the impurity finite-size scaling), one would obtain $\left\langle\hat{\sigma}_{1}^{x}\right\rangle=0$. To fix this point, in the following we adopt the method based on the asymptotic factorizability. Specifically, we compute $\left\langle\sigma_{1}^{x}\right\rangle$ at any finite $N$ as

$$
\left\langle\hat{\sigma}_{1}^{x}\right\rangle=\sqrt{\lim _{r \rightarrow \infty}\left\langle\hat{\sigma}_{1}^{x} \hat{\sigma}_{r}^{x}\right\rangle},
$$

which is equivalent [55] to

$$
\left\langle\hat{\sigma}_{1}^{x}\right\rangle \equiv\left|\left\langle E_{0}\left|\hat{\sigma}_{1}^{x}\right| E_{1}\right\rangle\right| \equiv\left|h_{1}^{(0)}\right|,
$$

with $\left|E_{0}\right\rangle$ and $\left|E_{1}\right\rangle$ being the ground and first excited states of the transverse field Ising Hamiltonian at given $N$ and $\mu$, respectively. Labeling the corresponding eigenfunctions as $\psi_{n}^{(0)}$ and $\phi_{n}^{(0)}$, as in Table I, one therefore obtains $h_{1}^{(0)}=\phi_{1}^{(0)}$. As a result, if $\left|E_{0}\right\rangle$ and $\left|E_{1}\right\rangle$ become degenerate as $N \rightarrow \infty$, giving rise to spontaneous symmetry breaking, a nonzero value for $\left\langle\hat{\sigma}_{1}^{x}\right\rangle$ occurs. On the contrary, if a finite-energy gap between the two states persists even as $N \rightarrow \infty$, so that no spontaneous symmetry breaking takes place, we have $\left\langle\hat{\sigma}_{1}^{x}\right\rangle=0$. It is worth noticing that Eq. (12) is actually valid only after the limiting procedure (in fact, the exponent $\beta$ computed in Refs. [39,56] refers to the critical scaling in a semi-infinite chain: no mention is made of finite-size scaling). Let us point out that Eq. (12) makes sense only if the edge spins are exchangeable. While this is clearly true for the homogeneous transverse field Ising model, it does not apply to our system at $\mu \neq 1$. Therefore, in order to correctly evaluate $\left\langle\hat{\sigma}_{1}^{x}\right\rangle$ with Eq. (12), in performing the calculation at finite $N$, we consider a mirror-symmetric version of our system, that is, we modify $h_{n}$ to $\tilde{h}_{n}$, given by

$$
\tilde{h}_{n}=h\left(1-\delta_{n, 1}\right)\left(1-\delta_{n, N}\right)+\mu h\left\{\delta_{n, 1}+\delta_{n, N}\right\}
$$

with a second, symmetric impurity at the end of the chain. Apparently, this should yield the appropriate result for our impurity system, provided one takes a large enough $N$. Taking all the above caveats into account, and performing the calculation using the wave functions in Table I, one eventually obtains that, as $N \rightarrow \infty$, the longitudinal impurity magnetization is given by

$$
\left\langle\hat{\sigma}_{1}^{x}\right\rangle=\sqrt{\frac{1-h^{2}}{1+h^{2}\left(\mu^{2}-1\right)}} \Theta(1-h) .
$$

Equation (15) shows that, as expected, one gets $\left\langle\hat{\sigma}_{1}^{x}\right\rangle=0$ for $h \geqslant 1$. At the same time, for $h \rightarrow 1^{-}$, one obtains to leading order

$$
\left\langle\hat{\sigma}_{1}^{x}\right\rangle \simeq \frac{\sqrt{2(1-h)}}{|\mu|} .
$$

From Eq. (16) we infer that, on one hand, a value of $\mu<1$ gives rise to an effective renormalization of the magnetic moment of the impurity spin [52]. On the other hand, and more importantly, the critical index $\beta=\frac{1}{2}$ obtained for the boundary magnetization in the homogeneous open boundary transverse field Ising model $[56,57]$ is not affected for $\mu \neq 1$.

We now move to discussing the impurity finite-size scaling in our inhomogeneous transverse field Ising model.

\section{FINITE-SIZE IMPURITY SCALING}

The idea of finite size scaling [58] is based on the observation that the behavior of a finite-size statistical system of typical size $\sim a N$, with $a$ being the lattice step (we set $a=1$ throughout this paper), is determined by a scaled variable $x=\frac{N}{\xi}$, with $\xi$ being the correlation length. As scaling is a property of large-size (i.e., thermodynamical) systems, one expects it to be recovered only over a scale $x$ such that $x \ll 1$. 
In this section, we discuss finite-size scaling of the impurity magnetization (transverse and longitudinal) as well as of the transverse susceptibility eventually showing how, and for which values of the system parameters, on varying the size $N$, the corresponding data for the various impurity observables collapse onto each other, with scaling exponents consistent with those derived in Sec. III in the thermodynamic limit. Specifically, we first compute the various impurity-related quantities at a finite system size $N$. Then, by fitting the results obtained in this way with the standard finite-size scaling formulas, we extract the correlation length $\xi(h)$ associated to the bulk quantum phase transition as a function of $h$. We find that $\xi(h) \sim\left|h-h_{c}\right|^{-v}$, with $v=1$. Thus, when either $h \ll h_{c}$ or $h \gg h_{c}$ (that is, far enough from the quantum phase transition), one finds that $\xi(h) \ll N$. Accordingly, in this regime the various observable quantities do not depend on $N$ and their values correspond to what one finds in the thermodynamic limit. On the other hand, by approaching the quantum phase transition $\xi(h) \simeq N$ and, consequently, the observables take a finite-size dependence on $N$, which is the key point of our scaling analysis $[42,43]$.

We now start to perform the finite-size scaling analysis for the transverse impurity magnetization close to the quantum phase transition. According to the derivation of Sec. III, we expect $\left\langle\hat{\sigma}_{1}^{z}\right\rangle$ to exhibit scaling only for $|\mu| \ll 1$. In this regime, the finite-size scaling ansatz for $\left\langle\hat{\sigma}_{1}^{z}\right\rangle$ gives

$$
\left\langle\hat{\sigma}_{1}^{z}\right\rangle \sim N^{-\frac{\hat{\beta}}{v}} f\left(N^{\frac{1}{v}}\left|h-h_{c}^{\prime}\right|\right),
$$

with the critical exponents $\tilde{\beta}$ and $v$ describing the singular behavior of the transverse impurity magnetization and of the correlation length, respectively, and with $f(x)$ being a suitable scaling function. According to Eq. (17), we determine the ratio $\frac{\tilde{\beta}}{v}$ by plotting the rescaled magnetization $N^{\frac{\beta}{\tilde{\beta}}}\left\langle\hat{\sigma}_{1}^{z}\right\rangle$ versus $h$. In order to do so, recalling that, for $\mu \rightarrow 0$, only the $\Lambda_{2}$ mode contributes to $\left\langle\hat{\sigma}_{1}^{z}\right\rangle$ [see Eqs. (5) and (6)], we have to employ the finite- $N$ version of the functions $\psi_{n}^{(2)}$ and $\phi_{n}^{(2)}$ of Table I, which, for $\mu=0$, are

$$
\psi_{n}^{(2)}=\sqrt{\frac{h^{2}-1}{1-h^{-2 N}}} h^{-n}, \quad \phi_{n}^{(2)}=\delta_{n 1} .
$$

This implies

$$
\left\langle\hat{\sigma}_{1}^{z}\right\rangle=\frac{1}{2 h} \sqrt{\frac{h^{2}-1}{1-h^{-2 N}}} .
$$

To first order in $\left(h-h_{c}\right)$, Eq. (19) gives

$$
\left\langle\hat{\sigma}_{1}^{z}\right\rangle \approx N^{-\frac{1}{2}}\left(\frac{1}{2}+\frac{N-1}{4}(h-1)\right) .
$$

From Eq. (19) for $n=1$, we construct the plot reported in the upper left inset of Fig. 3, where, in particular, we show that the curves representing the rescaled magnetization versus $h$ intersect with each other at $h=h_{c}=1$ once one sets $\frac{\tilde{\beta}}{v}=\frac{1}{2}$. Having determined $\frac{\tilde{\beta}}{v}$, we can proceed to the estimation of $v$ from the ansatz in Eq. (17) by looking for the best exponent for which the data collapse onto a single curve. A sample of our results is shown in the lower-right inset of Fig. 3. From such an analysis, we obtain $v=1$ and, therefore, $\tilde{\beta}=\frac{1}{2}$, a result
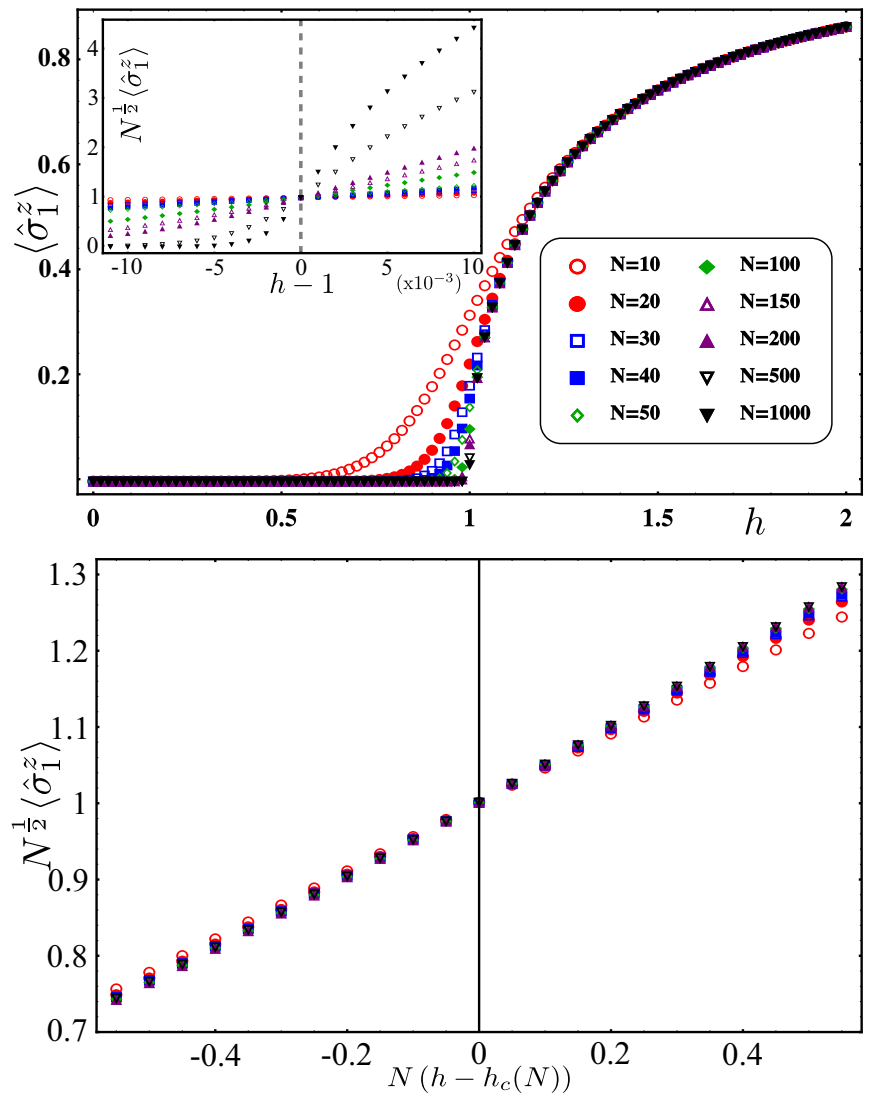

FIG. 3. Upper plot: transverse impurity magnetization $\left\langle\hat{\sigma}_{1}^{z}\right\rangle$ as a function of the bulk magnetic field $h$ for different system sizes $N$. Inset: finite-size scaling ansatz $N^{\frac{\tilde{\beta}}{v}}\left\langle\sigma_{0}^{z}\right\rangle$ versus $h$, with $\frac{\beta}{v}=\frac{1}{2}$. The intersection shows the location of the critical point at $h_{c}=1$. Lower plot: data collapse obtained with the scaling ansatz of Eq. (17).

that fully agrees with the critical exponent for $\left\langle\hat{\sigma}_{1}^{z}\right\rangle$ derived in Sec. III in the thermodynamic limit.

Next, we move on to discuss the finite-size scaling of the impurity transverse susceptibility. As shown in the main plot of Fig. $4, \chi_{z}^{(1)}$ diverges for $h \rightarrow 1$ in the thermodynamic limit. From the corresponding plots, we can fit the shift exponent $\lambda$ ruling the approach to $h_{c}$ of the pseudocritical value $h_{c}^{\prime}$ via the relation $\left|h_{c}^{\prime}-h_{c}\right| \propto N^{-\lambda}$. Our results are consistent with the value $\lambda=1$ (upper right inset of Fig. 4). Finally, from the relation $\lambda=\frac{1}{v}$, we again obtain $v=1$. Using the relation $\left.\chi_{z}^{(1)}\right|_{h=h_{c}^{\prime}} \sim N^{\frac{\alpha}{v}}$, we obtain for the ratio $\frac{\alpha}{v}$ a value compatible with $\frac{1}{2}$ (lower right inset of Fig. 4). Given the above results, we can now verify the consistency of the data collapse of $\chi_{z}^{(1)}$ with the finite-size scaling ansatz

$$
\chi_{z}^{(1)}(h)=N^{\frac{\alpha}{v}} \tilde{\chi}\left(N^{\frac{1}{v}}\left(h-h_{c}^{\prime}\right)\right)
$$

In the upper left inset of Fig. 4, we actually see such a data collapse for the predetermined values $v=1$ and for $\alpha=\frac{1}{2}$. Finally, in addition to the critical exponents for $\chi_{z}^{(1)}(h)$, and in analogy with the analysis for $\left\langle\hat{\sigma}_{1}^{z}\right\rangle$, we can also analytically determine the corresponding scaling. Indeed, from Eq. (19), 

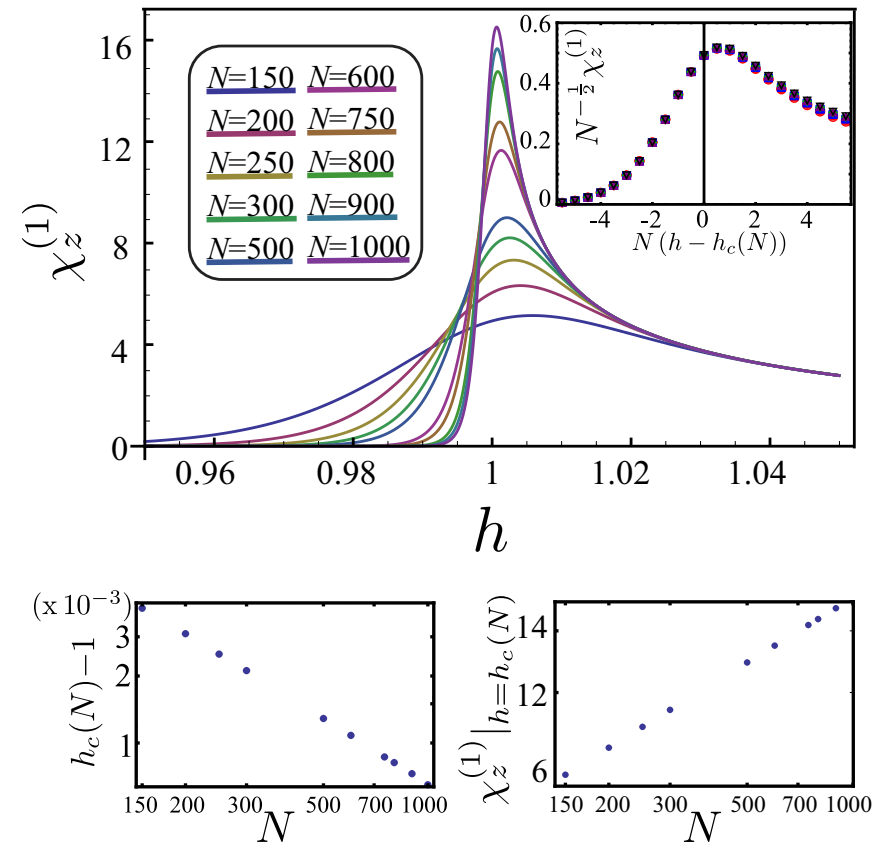

FIG. 4. Upper plot: impurity susceptibility $\chi_{z}^{(1)}$ for different system sizes, in the vicinity of $h=1$, where a diverging behavior is clearly displayed. Inset: data collapse for the impurity transverse susceptibility obtained with scaling exponents $v=1$ and $\alpha=\frac{1}{2}$. Lower left plot: log-log plot of the pseudocritical point $h_{c}(N)$ vs $N$, obtained from the position of the maximum of $\chi_{z}^{(1)}$, which allows to extract the shift exponent $\lambda$. Lower right plot: plot of the maximum of $\chi_{z}^{(1)}$ vs $N$ which shows the logarithmic nature of the divergence reported in the upper plot.

we obtain

$$
\chi_{z}^{(1)}=\frac{d\left\langle\hat{\sigma}_{1}^{z}\right\rangle}{d h}=\frac{h^{2(N-1)}\left[N\left(1-h^{2}\right)+h^{2 N}-1\right]}{2\left(h^{2 N}-1\right)^{2} \sqrt{\frac{h^{2 N}\left(h^{2}-1\right)}{h^{2 N}-1}}} .
$$

Expanding Eq. (22) for $h \simeq 1$ and for $N \gg 1$, we eventually find

$$
\chi_{z}^{(1)} \sim N^{\frac{1}{2}}\left[\frac{1}{4}+\left(\frac{N}{12}-1\right) \frac{h-1}{2}\right] .
$$

Equation (23) states that the critical exponent for the impurity transverse susceptibility is $\frac{1}{2}$, as well. From Eq. (22), we can also determine the position of the maximum, obtaining, once again, the shift of the pseudocritical point as $h_{c}^{\prime}-1 \simeq \frac{2}{3} N^{-1}$. This indicates that the shift exponent is $\lambda=1=\frac{1}{v}$, in full agreement with previous theoretical [58] and numerical [59] predictions.

To summarize, so far we have performed a finite-size scaling analysis of the impurity-related observables close to the quantum phase transition, obtaining the following critical exponents: $v=1, \tilde{\beta}=\frac{1}{2}$, and $\alpha=\frac{1}{2}$. It is important to stress once more that the condition $\mu=0$ is crucial, in order to witness the quantum phase transition. Finally, let us remark that the susceptivity of the homogeneous Ising model, corresponding to the specific heat in the two-dimensional (2D) classical Ising, has a logarithmic divergence $\left.\chi_{z}\right|_{h=h_{m}} \sim$ $\log N$, and the critical exponent is $\alpha=0$. As a consequence, the impurity susceptibility signals a qualitatively different phenomenon occurring at the system's edge. We now move on to consider the finite-size scaling of the longitudinal magnetization $\left\langle\hat{\sigma}_{1}^{x}\right\rangle$.

First of all, in order to recover an (approximate) analytic counterpart of the numerical results we discuss in the following, we have to properly generalize Eq. (15) to a finite $N$. Using again the wave functions listed in Table I, it is not difficult to show that, for finite $N$ and for $h \rightarrow 1^{-}$, one obtains

$$
\begin{aligned}
\left\langle\hat{\sigma}_{1}^{x}\right\rangle= & \sqrt{\frac{1-h^{2}}{1+\left(\mu^{2}-1\right) h^{2}}} \\
& \times\left\{1+\frac{\mu^{2}\left(1-h^{2}\right)}{1+\left(\mu^{2}-1\right) h^{2}} N h^{2 N}+O\left(N h^{2 N}\right)\right\} .
\end{aligned}
$$

As a preliminary check, in Fig. 5 we show the finite-size scaling data collapse for $\left\langle\hat{\sigma}_{1}^{x}\right\rangle$ as a function of $h$ in the absence of the impurity $(\mu=1)$. From the main plot in Fig. 5, as $N$ gets large, we recover the expected scaling of $\left\langle\hat{\sigma}_{1}^{x}\right\rangle$ as $(1-h)^{\frac{1}{2}}$ for $h<1$, as from Eq. (15) for $\mu=1$, while $\left\langle\hat{\sigma}_{1}^{x}\right\rangle$ experiences a steep decrease to zero for $h>1$ (disordered phase). To actually check finite-size scaling, in the bottom-left inset of Fig. 5, we plot $N^{\frac{\beta}{v}}\left\langle\hat{\sigma}_{1}^{x}\right\rangle$, for various values of $N$ (see figure caption for details). We find the expected intersection between all the curves drawn for various values of $N$ once we set $\beta=\frac{1}{2}, v=1$, consistently with Eq. (24) taken for $\mu=1$ and for $h \rightarrow 1^{-}$, as well as with the finite-size scaling hypothesis for a homogeneous chain. In fact, our result is further corroborated by the plot in the top-right inset of Fig. 5, where we draw $N^{\frac{\beta}{v}}\left\langle\hat{\sigma}_{1}^{x}\right\rangle$ for $\beta=\frac{1}{2}, v=1$, as a function of the rescaled variable $N^{\frac{1}{v}}\left[h-h_{c}(N)\right]$, finding an excellent data collapse, in agreement with finite-size scaling analysis based on the scaling formula for $\left\langle\hat{\sigma}_{1}^{x}\right\rangle$, given by

$$
\left\langle\hat{\sigma}_{1}^{x}\right\rangle=N^{-\frac{\beta}{v}} g\left(\left(h-h_{c}\right) N^{\frac{1}{v}}\right),
$$

with $\beta=\frac{1}{2}, v=1$. For $\mu \neq 1$, the behavior of the longitudinal magnetization changes substantially. Indeed, from Fig. 6, Eq. (25) appears to be definitely violated if $\mu<1$. On the other hand, as soon as $N$ gets large enough, Eq. (15) fits well the numerical data at any $\mu$. A possible explanation of such a finite-size scaling violation can be recovered by combining the results for the phase diagram in Fig. 1 with the finite- $N$ approximate formula for $\left\langle\hat{\sigma}_{1}^{x}\right\rangle$ in Eq. (24). From Fig. 1, one sees that on varying $h$ from left to right at a fixed $\mu$, one actually encounters two phase transitions: a first one, corresponding to the closure of the bulk Ising ordered phase at $h \sim 1$, and a second one, corresponding to the appearance of the mode $\Lambda_{2}$ at $|h(1-\mu)| \geqslant 1$. The former (ferromagnetic-paramagnetic) quantum phase transition is a bulk phenomenon, that is, it affects the longitudinal magnetization at any point of the open Ising chain, though with different exponents in the bulk and at the boundary. The latter transition, instead, has to be rather regarded as an impurity phase transition, not accompanied by a change in the bulk phase of the system. Nevertheless, in analogy with the emergence of a dynamically generated Kondo length [12], a length scale $\xi_{\mu}$ different from $\xi(h)$ seems to appear near this second transition, with eventually $\xi_{\mu} \rightarrow \infty$ right at the critical point. An approximate explicit formula for $\xi_{\mu}$ valid for $h \sim 1$ can be inferred from Eq. (24), which can be 

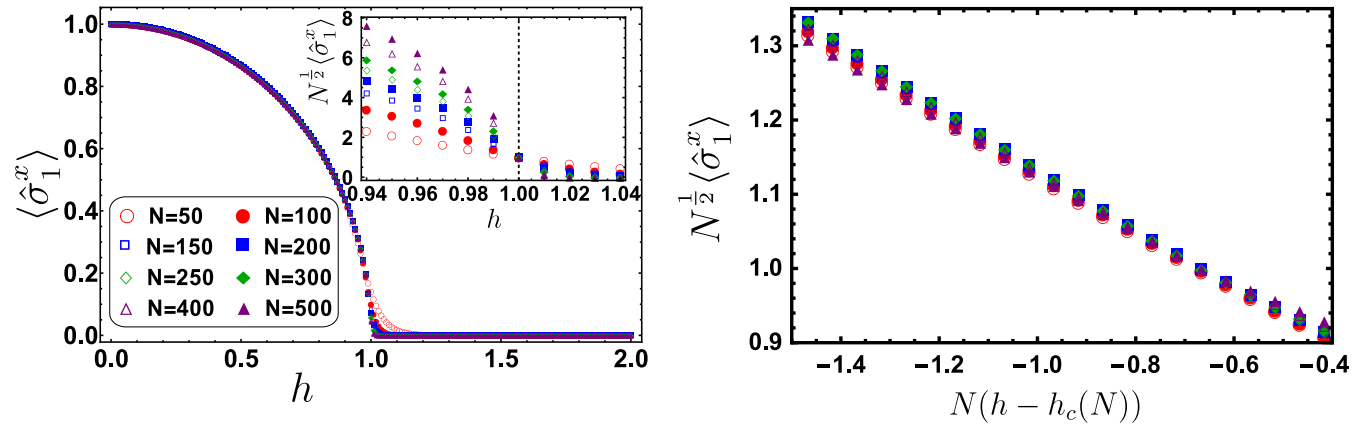

FIG. 5. Left plot: longitudinal impurity magnetization $\left\langle\hat{\sigma}_{1}^{x}\right\rangle$ as a function of $h$ for the homogeneous finite-size spin chain $(\mu=1)$, evaluated according to Eq. (12). Inset: zoom around the critical point of $N^{\frac{\beta}{v}}\left\langle\hat{\sigma}_{1}^{x}\right\rangle$ as a function of $h$, with $\beta=\frac{1}{2}, v=1$. Right plot: data collapse according to the finite-size scaling relation in Eq. (25) with $\beta=\frac{1}{2}, v=1$.

rewritten as

$$
\left\langle\hat{\sigma}_{1}^{x}\right\rangle=\frac{1}{\mu} \sqrt{\frac{1}{\xi(h)+\xi_{\mu}}}\left\{1+\frac{N}{\xi(h)+\xi_{\mu}} e^{-\frac{N}{\xi(h)}}\right\},
$$

with $\xi_{\mu}=\mu^{2} /\left|1-\mu^{2}\right|$. Equation (26) provides a possible explanation of the scaling violation for $\mu \in(0,1)$. Indeed, when comparing it with the generic finite-size scaling ansatz formula in Eq. (25), we see that in order for the two of them to be consistent with each other, Eq. (25), which can be recast in the form $\left\langle\hat{\sigma}_{1}^{x}\right\rangle=N^{-\frac{\beta}{v}} \tilde{g}\left(\frac{N}{\xi(h)}\right)$, must be generalized to an expression of the general form

$$
\left\langle\hat{\sigma}_{1}^{x}\right\rangle=N^{-\frac{\beta}{v}} \phi\left[\frac{N}{\xi(h)}, \frac{N}{\xi_{\mu}}\right] .
$$

While it would be definitely of interest to corroborate the two-length scenario with, for instance, a detailed analytical derivation of the formula for $\xi_{\mu}(h)$ at a generic value of $h$, this would require a careful analytical study of the transverse field Ising model in the presence of a boundary impurity, which goes beyond the scope of this paper, focused on the discussion of the finite-size scaling in the transverse field Ising model on a finite chain. Therefore, while we plan to address in detail this issue in a forthcoming publication, here we limit ourselves to a few additional observations on the two-length generalized scaling formula in Eq. (27). First of all, we note that, as $\mu \rightarrow 1$ (that

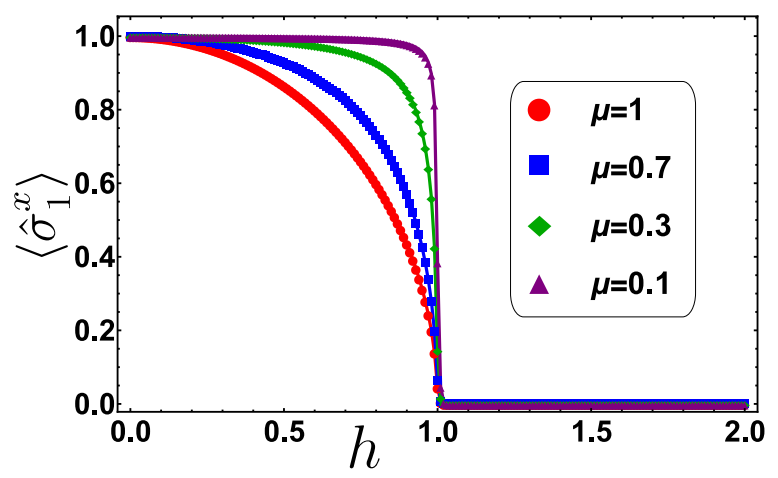

FIG. 6. Longitudinal impurity magnetization $\left\langle\hat{\sigma}_{1}^{x}\right\rangle$ for $N=500$ at different values of $\mu$. is, when going back to the homogeneous chain case), one gets $\xi_{\mu} \rightarrow \infty$, which implies that, in this limit, Eq. (27) reduces back to the standard, single-length finite-size scaling formula in Eq. (25), as it must be.

In the complementary limiting case $\mu \rightarrow 0$, we have $\xi_{\mu} \rightarrow$ 0 ; then, looking at Eq. (27), one would again expect a finite-size scaling formula such as in Eq. (25). In fact, this is quite a peculiar scaling limit in that, as $\mu \rightarrow 0$, one finds that $\hat{\sigma}_{1}^{x}$ becomes an exactly conserved quantity, even at finite $N$. In Fig. 7, we plot $\left\langle\hat{\sigma}_{1}^{x}\right\rangle$ as a function of $h$ for a rather small value of $\mu$ (the $\mu=0$ limit was hard to recover in the numerical calculations, due to the increasing lack of numerical precision for $\mu<0.1)$. Apparently, Fig. 7 is consistent with the previous discussion since $\left\langle\hat{\sigma}_{1}^{x}\right\rangle$ remains basically constant and finite, for $h<1$, while it suddenly jumps to 0 , as soon as $h$ crosses the quantum phase transition point. Looking back at Eq. (25), one may actually state that this behavior corresponds to an effective $\beta=0$, which appears to be consistent with $\hat{\sigma}_{1}^{x}$ being an exactly conserved quantity for $\mu=0$, at any value of $N$.

As a one-sentence summary of this section, one can state that, while the longitudinal magnetization $\left\langle\hat{\sigma}_{1}^{x}\right\rangle$ exhibits a neat finite-size scaling with $\beta=\frac{1}{2}, v=1$ at $\mu=1$, with increasing deviation from this behavior as $\mu$ moves from 1 to 0 , the transverse magnetization $\left\langle\hat{\sigma}_{1}^{z}\right\rangle$ shows a sort of fully complementary behavior: no scaling at any $\mu>0$, with an emerging scaling behavior recovered at $\mu=0$.

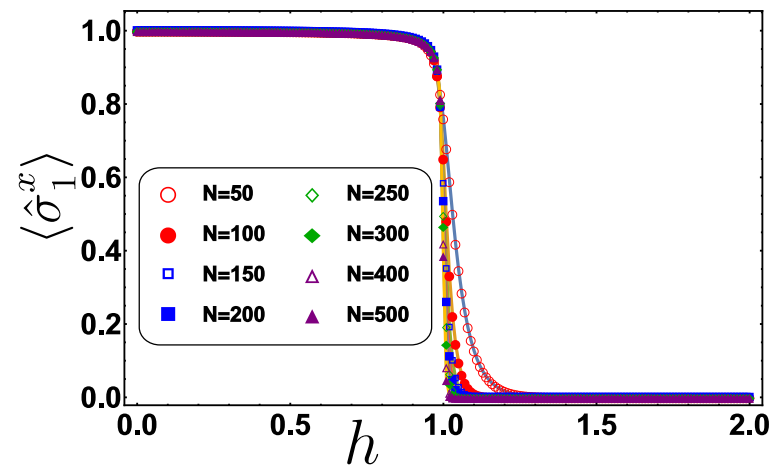

FIG. 7. Longitudinal impurity magnetization $\left\langle\hat{\sigma}_{1}^{x}\right\rangle$ for different values of $N$ evaluated for $\mu=0.1$. 


\section{FINITE-SIZE SCALING IN THE $X Y$ MODEL}

In this section, we test the universality of the impurity magnetic observables scaling properties by extending the previous analysis to the $X Y$ model, which belongs to the same universality class as the Ising model. To this end, we consider the following model Hamiltonian, describing a side impurity in the $X Y$ chain,

$$
\begin{aligned}
\hat{H}_{\mu}^{X Y}= & -J\left[\sum_{n=1}^{N-1}\left\{\left(1+\gamma_{n}\right) \hat{\sigma}_{n}^{x} \hat{\sigma}_{n+1}^{x}+\left(1-\gamma_{n}\right) \hat{\sigma}_{n}^{y} \hat{\sigma}_{n+1}^{y}\right\}\right. \\
& \left.+\sum_{n=1}^{N} h_{n} \hat{\sigma}_{n}^{z}\right],
\end{aligned}
$$

with the side impurity at $n=1$ defined by setting

$$
\begin{aligned}
& \gamma_{n}=\gamma\left(1-\delta_{n 1}\right)+\gamma_{1} \delta_{1 n}, \\
& h_{n}=h\left(1-\delta_{n 1}\right)+\mu h \delta_{n 1} .
\end{aligned}
$$

This choice corresponds to both the magnetic field and the anisotropy parameter staying homogeneous on the bulk, with values $h$ and $\gamma$, but taking different values at the edge of the chain, where they become $\mu h$ and $\gamma_{1}$, respectively.

With the bulk anisotropy parameter $\gamma \in(0,1]$, the $X Y$ model undergoes a quantum phase transition at a critical value of $h=h_{c}$, which falls into the same universality class as the transverse field Ising model. Our aim here is to investigate whether the bulk equivalence extends to the impurity finite-size scaling, as well.

An analytical solution of the impurity model for $\gamma \neq 1$ analogous to that provided in Ref. [53] for the Ising model is lacking. Nevertheless, one may readily check that, as $\mu \rightarrow 0$, the parity operator $\hat{P}$ is again a good symmetry of the model, that is, $\left[\hat{H}_{X Y ; \mu=0}, \hat{P}\right]=0$, provided that $\gamma_{1}= \pm 1$ (which means an Ising-type coupling, either along the $X$ or the $Y$ direction). One can then analytically verify that both possibilities imply the presence of a zero-energy eigenvalue, in analogy to what happens in the transverse field Ising model in Eq. (1). It is hence reasonable to expect that, also in this case, one recovers the behavior of a classical edge impurity, just as in the Ising model. Indeed, we obtain, for the whole Ising universality class, $\gamma \in(0,1]$, the same finite-size scaling exponents as in the transverse field Ising model, that is, $v=1$, $\tilde{\beta}=\frac{1}{2}$, and $\alpha=\frac{1}{2}$. To highlight this result, in Fig. 8 we show the data collapse of the transverse impurity magnetization and susceptibility, according to Eqs. (17) and (21) for the $X Y$ model with bulk anisotropy $\gamma=0.5$.

\section{CLASSICAL-TO-QUANTUM CORRESPONDENCE IN THE ISING MODEL WITH AN IMPURITY}

The one-dimensional quantum Ising model can be mapped onto the two-dimensional classical model as a special case of the general correspondence between critical phenomena in $d$-dimensional quantum statistical models and those in $(d+1)$-dimensional classical statistical models. Such a correspondence has been largely discussed and reviewed in the literature, particularly in the context of lattice gauge theories and classical, as well as quantum, lattice spin models [40,41]. The classical two-dimensional Ising model is one of the

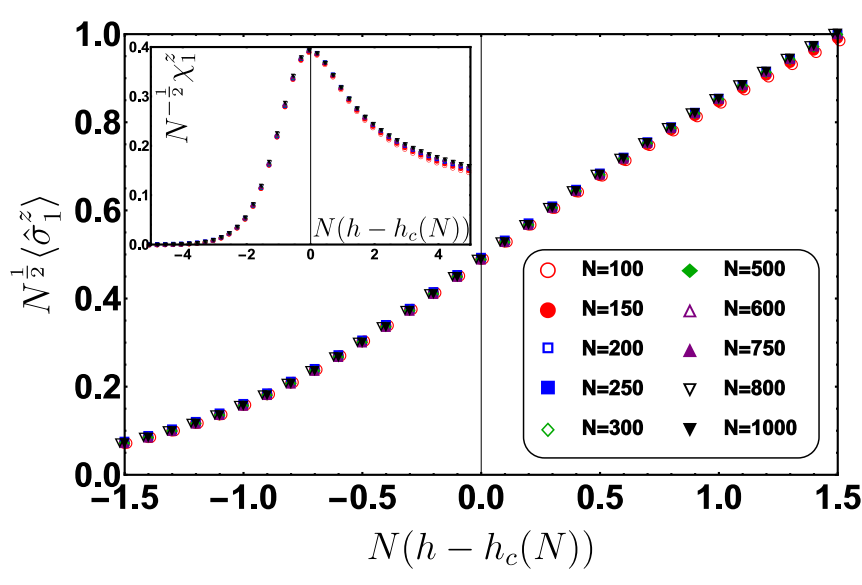

FIG. 8. Main plot: data collapse of the transverse impurity magnetization $\left\langle\hat{\sigma}_{1}^{z}\right\rangle$ for an $X Y$ model [Eq. (28)] with $\gamma=0.5$ and $\gamma_{1}=1$. Inset: transverse local spin susceptibility for the same value of $\gamma$. The critical exponents take the same values as in the plots in Figs. 3 and 4, respectively.

best-known classical statistical systems, especially (but not only) in view of the remarkable Onsager's exact solution of the model (see, for instance, Ref. [56] for an extensive review on the topic). According to this correspondence, one finds, e.g., that the transverse field Ising model with periodic boundary conditions is related to a $d=2$ classical Ising model on a torus [44], while the homogeneous transverse field Ising model with open boundary conditions corresponds to a finite-size cylinder whose boundary circles map onto the (quantum) end points of the chain $[39,56]$. One may generalize the mapping to the case of models with generic, position-dependent parameters as outlined in Ref. [60]. Following [60], in this section we construct the mapping between the inhomogeneous transverse field Ising model with an impurity at its left-hand side end point and the corresponding 2D classical spin model.

Following the approach discussed in Appendix B of Ref. [60], we choose as a reference 2D classical system the planar Ising model with inhomogeneous couplings, described by the Hamiltonian

$$
\begin{aligned}
\mathcal{E}\left[\left\{\sigma_{n, m}\right\}\right]= & -\sum_{n=1}^{M_{x}} \sum_{m=1}^{M_{y}} K_{y}(n) \sigma_{n, m} \sigma_{n, m+1} \\
& -\sum_{n=1}^{M_{x}-1} \sum_{m=1}^{M_{y}} K_{x}(n) \sigma_{n, m} \sigma_{n+1, m},
\end{aligned}
$$

with the couplings $K_{x}$ and $K_{y}$ generically depending on $n$ and with $\sigma_{n, m}$ being a classical (binary) spin variable at site $n, m$ of the lattice (which we shall identify with the $x$ component of the quantum spin when mapping to a $1 \mathrm{D}$ inhomogeneous transverse field Ising model) and with periodic boundary conditions assumed in the $y$ direction, that is, $\sigma_{n, m+M_{y}}=$ $\sigma_{n, m}, \forall n=1, \ldots, M_{x}$ (so that, pictorially, the lattice may be thought as wrapped around a cylinder along the $y$ direction). At temperature $T=\beta^{-1}$, one obtains the classical partition 
function for the model in Eq. (30) as

$$
\mathcal{Z}\left[\left\{K_{x}, K_{y}\right\} ; T\right]=\sum_{\left\{\sigma_{n, m}= \pm 1\right\}} \exp \left\{\beta\left[\sum_{n=1}^{M_{x}} \sum_{m=1}^{M_{y}} K_{y}(n) \sigma_{n, m} \sigma_{n, m+1}+\sum_{n=1}^{M_{x}-1} \sum_{m=1}^{M_{y}} K_{x}(n) \sigma_{n, m} \sigma_{n+1, m}\right]\right\} .
$$

To complete the mapping onto the 1D quantum Ising model, one has to to rewrite Eq. (31) as the partition function of a one-dimensional transverse field Ising model with nonhomogeneous parameters. This requires constructing an appropriate transfer matrix $\mathbf{T}$, whose matrix elements can be regarded as the partition function for two subsequent rows (say $m$ and $m+1)$ with assigned spin configurations $\left\{\left|\left\{\sigma_{n, m}\right\}_{n=1, \ldots M_{x}}\right\rangle_{m}\right.$ and $\left\{\left|\left\{\sigma_{n, m+1}^{\prime}\right\}_{n=1, \ldots M_{x}}\right\rangle_{m+1}\right\}$, so that

$$
[T]_{\left\{\sigma_{n}^{\prime}\right\},\left\{\sigma_{n}\right\}}={ }_{m+1}\left\langle\sigma_{n}^{\prime}|\mathbf{T}| \sigma_{n}\right\rangle_{m} .
$$

In the low- $T$ (large- $\beta$ ) limit, one may explicitly write $\mathbf{T}$ as [60]

$$
\mathbf{T}=\exp \left[-\hat{H}_{1 \mathrm{D}}\right],
$$

with $\hat{H}_{1 \mathrm{D}}$ being the Hamiltonian of the inhomogeneous quantum Ising chain given by (apart for an irrelevant overall constant term)

$$
\hat{H}_{1 \mathrm{D}}=-\sum_{n=1}^{M_{x}} h_{n} \hat{\sigma}_{n}^{z}-\sum_{n=1}^{M_{x}-1} J_{n} \hat{\sigma}_{n}^{x} \hat{\sigma}_{n+1}^{x}
$$

with

$$
\begin{aligned}
J_{n} & =\beta K_{x}(n), \\
e^{-2 h_{n}} & =\tanh \left[\beta K_{y}(n)\right],
\end{aligned}
$$

and $\hat{\sigma}_{n}^{x, z}$ being quantum spin operators on a 1D lattice.

Equation (35) gives the classical correspondent of the quantum Ising chain with an impurity at its leftmost site. In fact, one has to assume that Eq. (35) holds with $h_{n}$ and $K_{y}(n)$ independent of $n$ at any $n$ but for $n=1$. Basically, the classical model can be regarded as a cylinder that, as $N \rightarrow \infty$, goes all the way to $\infty$ towards the positive- $x$ axis. The cylinder is cut in correspondence of the first site of the quantum chain. The smaller is $h_{1}$, the stronger is the effective Ising coupling $\beta K_{y}(1)$ on the circumference running around the cut. Eventually, the limit of classical impurity in the chain $\left(h_{1} \rightarrow 0\right)$ corresponds to the limit $\beta K_{y}(1) \rightarrow \infty$, in which, consistently with what one expects from the classicalto-quantum correspondence, all the thermal fluctuations are suppressed in the classical Ising chain lying over the circle at $n=1$.

\section{CONCLUDING REMARKS}

In this paper, we have studied finite-size scaling for an impurity residing at the boundary (first site) of an otherwise homogeneous quantum Ising chain in a transverse magnetic field $h$, modeled by rescaling $h$ on the first site by $h \rightarrow h_{1}=\mu h$. To highlight finite-size scaling at the impurity, we computed in a finite-size chain (with $N$ sites) both the transverse and the longitudinal magnetization at the first site of the chain. This allowed us to single out the limits $\mu \rightarrow 1$ and $\mu \rightarrow 0$ as the two regimes in which "standard" finite-size scaling is recovered, though in complementary fashions. Indeed, while in the homogeneous chain limit $(\mu \rightarrow 1)\left\langle\hat{\sigma}_{1}^{x}\right\rangle$ exhibits finite-size scaling with $N^{-\frac{\beta}{v}}$, with $\beta=\frac{1}{2}$ and $v=1$, with $\left\langle\hat{\sigma}_{1}^{z}\right\rangle$ showing no finite-size scaling at all, in the complementary "classical impurity" limit $(\mu \rightarrow 0)$, full finite-size scaling is recovered for the transverse magnetization $\left\langle\hat{\sigma}_{1}^{z}\right\rangle$ with the same exponents reported above. On the other hand, the longitudinal magnetization $\left\langle\hat{\sigma}_{1}^{x}\right\rangle$ does not show scaling at $\mu=0$. This nicely corresponds to the fact that $\hat{\sigma}_{1}^{x}$ is an exactly conserved quantity in the finite chain, at $\mu=0$. In this limit, on extending the finite-size scaling analysis to the impurity transverse susceptibility $\chi_{z}^{(1)}(h)$, we have found again results consistent with the finite-size scaling ansatz and, in addition, have been able to recover the shift exponent governing the location of the quantum pseudocritical point at finite $N$.

Our analysis, thus, extends the finite-size scaling behavior of the homogeneous transverse field Ising model $[42,43]$ with open boundary conditions to the case in which an impurity is present at one end point of the chain by showing that, as $\mu=0$, the transverse surface magnetization $\left\langle\hat{\sigma}_{1}^{z}\right\rangle$ works as a sort of "disorder" parameter for the bulk (being nonzero in the disordered phase), with the same exponents characterizing the finite-size scaling of $\left\langle\hat{\sigma}_{1}^{x}\right\rangle$ for the homogeneous model $(\mu=1)$. As mentioned in the discussion of Sec. IV, the intermediate situation $0<\mu<1$ is much less clear, in that the scaling ansatz of Eq. (25) appears not to reproduce the finite-size behavior of $\left\langle\hat{\sigma}_{1}^{x}\right\rangle$. In fact, the smaller $\mu$, the more severe is the deviation of $\left\langle\hat{\sigma}_{1}^{x}\right\rangle$ from the general scaling formula. As outlined above, a possible explanation, consistent with the asymptotic expressions for $\left\langle\hat{\sigma}_{1}^{x}\right\rangle$ in Eqs. (15) and (24), relies on the emergence of two length scales, associated with the two QPTs the system goes through, for $\mu \neq 1$. While this appears to be a suggestive and promising hypothesis, its careful analytical and numerical verification goes beyond the scope of this work and, accordingly, we chose to postpone it to a forthcoming publication.

Finally, on employing the standard quantum-to-classical mapping for our specific impurity model, we have been able to construct the (two-dimensional) statistical model providing the classical counterpart of a transverse field Ising model with an impurity at one of its end points as a classical Ising model on a rectangular lattice wrapped on a half-infinite cylinder, with the links along the first circle altered, as a function of $\mu$, according to Eq. (35).

\section{ACKNOWLEDGMENTS}

We thank A. Nersesyan for useful correspondence and N. Lo Gullo, S. Lorenzo, A. Gambassi, and F. Franchini for insightful discussions. T.J.G.A., G.F., G.M.P., and F.P. acknowledge support from the Collaborative Project QuProCS (Grant Agreement No. 641277). 
[1] S. Sachdev, Quantum Phase Transitions, 2nd ed. (Cambridge University Press, Cambridge, 2011).

[2] G. Jaeger, Archive History Exact Sci. 53, 51 (1998).

[3] R. B. Griffiths, Phys. Rev. Lett. 24, 1479 (1970).

[4] L. P. Kadanoff, Physics 2, 263 (1966).

[5] J. Zinn-Justin, Quantum Field Theory and Critical Phenomena (Clarendon Press, Oxford, 2012).

[6] K. G. Wilson, Rev. Mod. Phys. 47, 773 (1975).

[7] S. Lorenzo, J. Marino, F. Plastina, G. M. Palma, and T. J. G. Apollaro, Sci. Rep. 7, 5672 (2017).

[8] A. Osterloh, L. Amico, G. Falci, and R. Fazio, Nature (London) 416, 608 (2002).

[9] S. Campbell, L. Mazzola, G. De Chiara, T. J. G. Apollaro, F. Plastina, T. Busch, and M. Paternostro, New J. Phys. 15, 043033 (2013).

[10] G. De Chiara, L. Lepori, M. Lewenstein, and A. Sanpera, Phys. Rev. Lett. 109, 237208 (2012).

[11] A. Bayat, T. J. G. Apollaro, S. Paganelli, G. De Chiara, H. Johannesson, S. Bose, and P. Sodano, Phys. Rev. B 93, 201106 (2016).

[12] I. Affleck, in Exact Methods in Low-dimensional Statistical Physics and Quantum Computing, edited by J. Jacobsen, S. Ouvry, V. Pasquier, D. Serban, and L. Cugliandolo (Oxford University Press, Oxford, 2004).

[13] S. Lorenzo, T. J. G. Apollaro, A. Sindona, and F. Plastina, Phys. Rev. A 87, 042313 (2013).

[14] S. Lorenzo, T. J. G. Apollaro, S. Paganelli, G. M. Palma, and F. Plastina, Phys. Rev. A 91, 042321 (2015).

[15] J. Kondo, Prog. Theor. Phys. 32, 37 (1964).

[16] A. C. Hewson, The Kondo Effect to Heavy Fermions (Cambridge University Press, Cambridge, 1993).

[17] A. Furusaki and T. Hikihara, Phys. Rev. B 58, 5529 (1998).

[18] N. Laflorencie, E. S. Sørensen, and I. Affleck, J. Stat. Mech.: Theor. Exp. (2008) P02007.

[19] C. L. Kane and M. P. A. Fisher, Phys. Rev. B 46, 15233 (1992).

[20] D. Giuliano and P. Sodano, Nucl. Phys. B 837, 153 (2010).

[21] D. Giuliano and P. Sodano, Europhys. Lett. 88, 17012 (2009).

[22] A. Cirillo, M. Mancini, D. Giuliano, and P. Sodano, Nucl. Phys. B 852, 235 (2011).

[23] D. Giuliano and P. Sodano, Nucl. Phys. B 770, 332 (2007).

[24] A. M. Tsvelik, Phys. Rev. Lett. 110, 147202 (2013).

[25] A. Altland, B. Béri, R. Egger, and A. M. Tsvelik, Phys. Rev. Lett. 113, 076401 (2014).

[26] A. M. Tsvelik, New J. Phys. 16, 033003 (2014).

[27] D. Giuliano, P. Sodano, A. Tagliacozzo, and A. Trombettoni, Nucl. Phys. B 909, 135 (2016)

[28] D. Giuliano, G. Campagnano, and A. Tagliacozzo, Eur. Phys. J. B 89, 251 (2016).

[29] S.-J. Ran, C. Peng, G. Su, and M. Lewenstein, arXiv:1707.07838.
[30] M. Knap, A. Shashi, Y. Nishida, A. Imambekov, D. A. Abanin, and E. Demler, Phys. Rev. X 2, 041020 (2012).

[31] M. Schirò and A. Mitra, Phys. Rev. Lett. 112, 246401 (2014).

[32] A. Sindona, J. Goold, N. Lo Gullo, S. Lorenzo, and F. Plastina, Phys. Rev. Lett. 111, 165303 (2013).

[33] A. Sindona, N. Lo Gullo, J. Goold, and F. Plastina, New J. Phys. 16, 045013 (2014).

[34] T. J. Elliott and T. H. Johnson, Phys. Rev. A 93, 043612 (2016).

[35] M. T. Mitchison, T. H. Johnson, and D. Jaksch, Phys. Rev. A 94, 063618 (2016).

[36] D. Tamascelli, C. Benedetti, S. Olivares, and M. G. A. Paris, Phys. Rev. A 94, 042129 (2016).

[37] M. Streif, A. Buchleitner, D. Jaksch, and J. Mur-Petit, Phys. Rev. A 94, 053634 (2016).

[38] F. Cosco, M. Borrelli, F. Plastina, and S. Maniscalco, Phys. Rev. A 95, 053620 (2017).

[39] I. Peschel, Phys. Rev. B 30, 6783 (1984).

[40] E. Fradkin and L. Susskind, Phys. Rev. D 17, 2637 (1978).

[41] J. B. Kogut, Rev. Mod. Phys. 51, 659 (1979).

[42] J. Um, S.-I. Lee, and B. J. Kim, J. Korean Phys. Soc. 50, 285 (2007).

[43] T. W. Burkhardt and I. Guim, J. Phys. A: Math. Gen. 18, L33 (1985).

[44] E. Lieb, T. Schultz, and D. Mattis, Ann. Phys. (NY) 16, 407 (1961).

[45] E. Barouch and B. M. McCoy, Phys. Rev. A 3, 786 (1971).

[46] L. Onsager, Phys. Rev. 65, 117 (1944).

[47] C. N. Yang, Phys. Rev. 85, 808 (1952).

[48] A. Dutta, G. Aeppli, B. K. Chakrabarti, U. Divakaran, T. F. Rosenbaum, and D. Sen, Quantum Phase Transitions in Transverse Field Spin Models: From Statistical Physics to Quantum Information (Cambridge University Press, Cambridge, 2015).

[49] R. B. Griffiths, Phys. Rev. Lett. 23, 17 (1969).

[50] T. Vojta, Phys. Rev. Lett. 90, 107202 (2003).

[51] T. Vojta, J. Phys.: Conf. Ser. 529, 012016 (2014).

[52] M. Müller and A. A. Nersesyan, Ann. Phys. 372, 482 (2016).

[53] G. Francica, T. J. G. Apollaro, N. Lo Gullo, and F. Plastina, Phys. Rev. B 94, 245103 (2016).

[54] P. Jordan and E. Wigner, Z. Phys. 47, 631 (1928).

[55] T. D. Schultz, D. C. Mattis, and E. H. Lieb, Rev. Mod. Phys. 36, 856 (1964).

[56] B. McCoy and T. Wu, The Two-dimensional Ising Model (Harvard University Press, Cambridge, MA, 1973).

[57] P. Pfeuty, Ann. Phys. (NY) 57, 79 (1970).

[58] C. Domb, M. Green, and J. Lebowitz, Phase Transitions and Critical Phenomena, Vol. 8 (Academic Press, New York, 1983).

[59] F. Iglói and Y.-C. Lin, J. Stat. Mech. (2008) P06004.

[60] F. Iglói, I. Peschel, and L. Turban, Adv. Phys. 42, 683 (1993). 